\title{
Brillouin distributed fiber sensors: practical limitations and guidelines for the making of a good sensor
}

\author{
Luc Thévenaz, Marcelo A. Soto \\ Ecole Polytechnique Fédérale de Lausanne \\ Institute of Electrical Engineering, SCI-STI-LT Station 11 \\ 1015 Lausanne, Switzerland \\ Email : luc.thevenaz@epfl.ch
}

\begin{abstract}
By analyzing the fundamentals of the operation of Brillouin sensors it will be shown that all efforts must be focused on obtaining the largest signal-to-noise ratio on the raw optical signal, which ultimately scales all performance of the sensor. Guidelines will be presented, together with recent research efforts to find out smart solutions pushing further the limits.
\end{abstract}

Keywords- Fiber optics sensors; distributed fiber sensors; stimulated Brillouin scattering.

\section{INTRODUCTION}

For the design of a good sensor all efforts must be focused on obtaining the largest signal-to-noise ratio on the raw optical signal. It can be shown that signal-to-noise ratio ultimately scales all performance of the sensor: distance range, spatial resolution, accuracy on temperature or strain, and acquisition speed [1]. Logically the making of a good sensor requires strategies to maximize the signal-to-noise ratio and guidelines will be presented, which consist essentially in generating the largest possible response of the sensor. Actually this response cannot be made arbitrarily large, since the maximum power of the interacting signals is limited by spectral-jamming nonlinear effects [2]. Avoiding too large pump depletion is also a crucial design point [3] and recent research efforts are oriented in finding smart solutions to push further the limits.

\section{DESCRIPTION OF THE PROBLEMATIC}

The ever growing demand for better specifications for this kind of sensor has recently stimulated efforts to boost the performance, particularly in terms of distance range, spatial resolution and acquisition speed. The response of the sensor is essentially proportional to the power of both interacting signals (see Fig. 1) that inevitably decay as a result of the linear loss [1].

In a small gain approximation that is always justified in high performance sensor (gain $\sim 1 \%$ and smaller) the response of a BOTDA sensor $\Delta P_{s}(z)$ is expressed as [1]:

$$
\Delta P_{s}(z)=\frac{g_{B}(z)}{A_{\text {eff }}} P_{P i} \exp (-\alpha z) P_{s i} \exp (-\alpha L) \Delta z
$$

where $g_{B}(z)$ represents the local Brillouin gain coefficient, $A_{\text {eff }}$ is the nonlinear effective area of the guided mode and $\Delta z$ is the interaction length, which is equivalent to the spatial resolution and determined by the pump pulse half duration multiplied by the group velocity of the guided mode. $P_{P i}$ and $P_{s i}$ are the input pump and probe powers, respectively, and $\alpha$ the linear attenuation coefficient in the fiber.

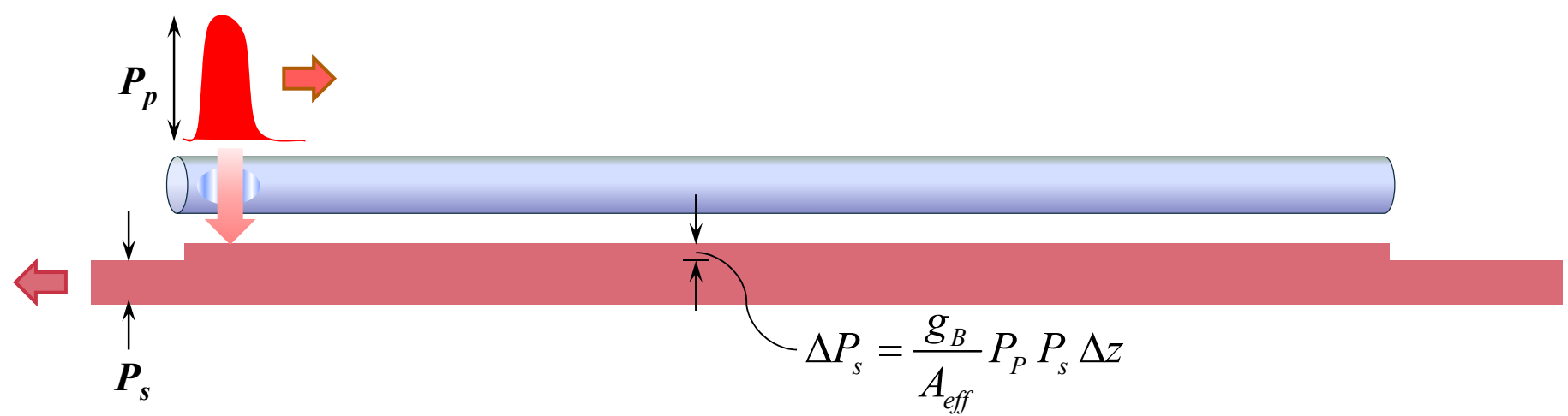

Fig. 1. Interacting signals in a generic BOTDA sensor. A light pulse of power $P_{P}-$ called pump - interacts with a continuous wave of power $P_{S}-$ called probe or signal - through the intercession of an idler acoustic wave if a strict phase matching condition is satisfied. Phase matching depends on the frequency difference between pump and probe and gives rise to a local power transfer $\Delta P_{s}$ between the interacting optical waves. $\Delta P_{s}$ represents the response of the sensor and must be maximized. 
A natural approach to get the largest response is to raise the input power of the interacting signals to compensate the unescapable loss due to linear attenuation, but limits are rapidly reached due to nonlinear effects depleting the interacting signals. More concretely, for the pump pulse, only copropagating nonlinearly generated waves can interact with the pump pulse over a long distance, so that the actual limiting effects are modulation instability (for peak pulse power $>100 \mathrm{mw}$ ) and forward Raman scattering (for peak pulse power $>500 \mathrm{mw}$ ) [2]. Modulation instability can be suppressed by propagating in a normal dispersion regime in a dispersion shifted fiber, while forward Raman scattering can never be totally suppressed.

The CW probe power is in addition subject to contrapropagating nonlinearly generated waves and is therefore limited by amplified spontaneous Brillouin scattering for probe power $>5 \mathrm{~mW}$. It must also be pointed out that the $\mathrm{CW}$ probe wave induces depletion on the pump pulse through the continuous power transfer due to the sensing interaction and this sets an even stricter limitation for the probe power that must be kept below $40 \mu \mathrm{W}$ [3]. Nevertheless solutions have been proposed to make the sensing system more robust to depletion, such as using a double probe wave in a spectrally symmetric situation with compensating depletion-amplification on the pump pulse $[3,4]$ or using the gain phase response that turns out to be very insensitive to pump power [5], so that the practical limit is practically given by amplified spontaneous Brillouin scattering in a properly designed sensor.

The number of resolved points is an important metric to evaluate how good a sensor is [6]. It is given by the ratio of the sensor distance range over the spatial resolution. This sets a stringent limit on the extinction ratio of the pump pulse, which must be roughly equivalent to the inverse of the number of resolved points, i.e. $10^{\prime} 000$ resolved points requires a $40 \mathrm{~dB}$ on/off ratio on the pump pulse. This condition comes from the fact that the $\mathrm{CW}$ light leaking through the pulse modulating device must give a Brillouin amplification integrated all over the fiber length that must be kept smaller than the local pulse amplification [7]. A high extinction ratio pulse shaping device is therefore normally required, which must be carefully selected and is not necessarily a standard off-the-shelf type.

For a given signal-to-noise ratio (SNR) on the detector the uncertainty on the final measurement can be entirely predicted thanks to a model that was rigorously compared with experimental data [1]. If $\Delta v_{B}$ is the estimated Brillouin fullwidth at half maximum (FWHM) of the resonance and $\delta$ is the frequency sampling step (i.e. the frequency increment between successive Brillouin intensity traces), the propagated error on the estimated resonance central frequency $\sigma_{v}$ expresses as:

$$
\sigma_{v}(z)=\frac{1}{S N R(z)} \sqrt{\frac{3}{4} \delta \cdot \Delta v_{B}} .
$$

The validity of this expression is subject to the condition $\delta<<\Delta v_{B}$, which is equivalent to require a large number of frequency sampling points. Fig. 2 shows the measured statistical error on the determination of the Brillouin peak gain

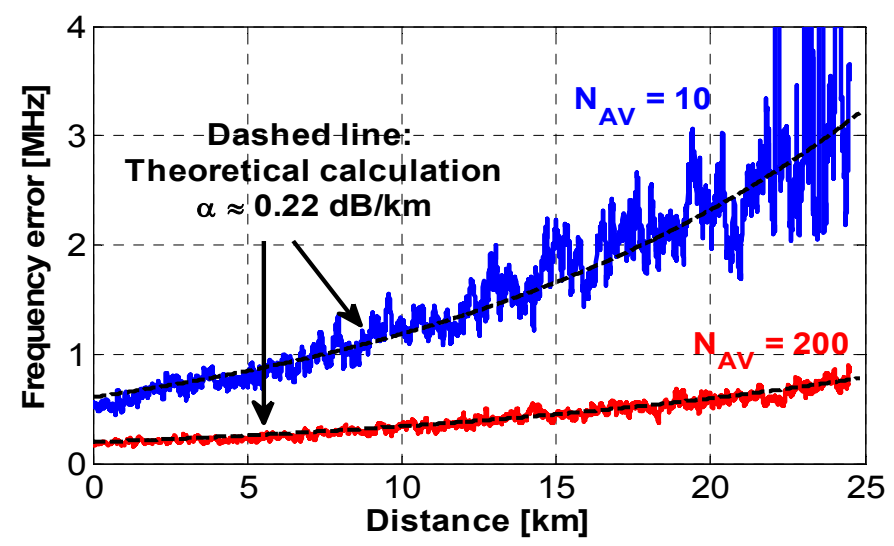

Fig.2. Frequency error on the peak Brillouin gain frequency as a function of distance distance, measured for 10 and 200 time-averaged traces (blue and red solid lines). Dashed lines: theoretical prediction given by the sensor model for a linear attenuation $\alpha=0.22 \mathrm{~dB} / \mathrm{km}[1]$.

frequency as a function of the position along the fiber, demonstrating a very good matching with the model.

The error on the final measurement can be arbitrarily reduced by increasing the number of averaging - that will make the SNR larger - or by increasing the number of sampling frequencies to determine the Brillouin gain spectrum, with equal impact on the final result. But this will inevitably be at the expense of a longer measurement time. A $10 \mathrm{~dB}$ SNR increase requires 100 times more averaging that will extend the measurement time in the same proportion. It can be easily concluded that optimizing the configuration to originally get a SNR as low as possible is always beneficial.

\section{POSSIBLE APPROACHES}

Within this strict framework smart solutions have been proposed to overcome these limits. A very clever approach uses schemes employing multiple pulses arranged in coded sequences to increase the interactive energy, while maintaining the peak power below the nonlinear limits [8]. This requires a coding procedure to determine the pulse sequence and the equivalent response for a single summed-up pulse can be retrieved through a linear transformation. Fig. 3 illustrates the very substantial gain in SNR obtained using a 511-bits Simplex coding, with no penalty in measurement time.

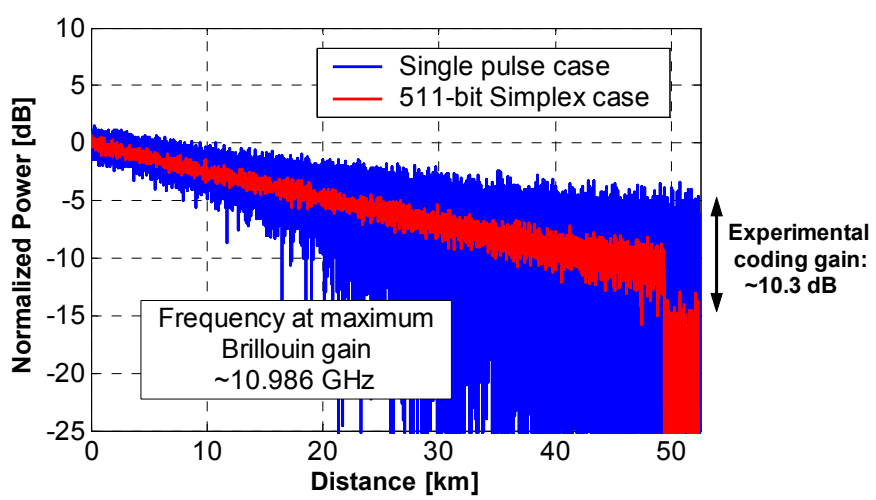

Fig. 3. Raw traces of the optical response of the sensor, for a single pulse (blue) and for a sequence of 511 pulses ordered using the Simplex coding. The SNR gain is more than $10 \mathrm{~dB}$ for an equivalent measuring 
Another solution is to use distributed amplification to compensate loss, using Raman pumping at each fiber ends, for instance [9]. This maintains an observable gain over an extended distance range, as illustrated in Fig. 4. This solution presents unfortunately the penalty to induce an important added noise.

The regeneration of the interacting signals by using repeaters based on erbium-doped fiber amplifiers is another interesting approach, similar to the regeneration of optical signals in telecommunication fiber links. It actually turns out to be not that straightforward, since the repeaters must solely amplify the pump and the probe and not the noise feeding modulation instability for the pump and amplified spontaneous Brillouin scattering for the probe. Since these seeding noise are in the direct spectral vicinity of the pump and the probe, it requires a smart design and an interesting solution has been recently proposed demonstrating a sensing range up to $325 \mathrm{~km}$ [10].

One of the most efficient solution is to spread the energy in the pump wave over multiple pulses, each at a different frequency [11], so that the peak power of each pulse is maintained below the nonlinear limits and the responses due to individual pulses can be summed up. The implementation is not straightforward, because the pulses must be shifted temporally to present no temporal overlapping, avoiding a mutual seeding of their modulation instability gain band and four wave mixing. Fig. 5 compares this case with a standard implementation [11], showing a substantial increase of the SNR without extending the measurement time and using a standard detection scheme.

All these solutions are mutually compatible and many combinations have already been demonstrated. Their ultimate finality is to eventually increase the amplitude of the raw detected optical signal to make the signal-to-noise ratio larger. A future approach would be to drastically reduce the optical noise at the light source instead of making the response larger, for a similar effect. This has been so far still widely unexplored.

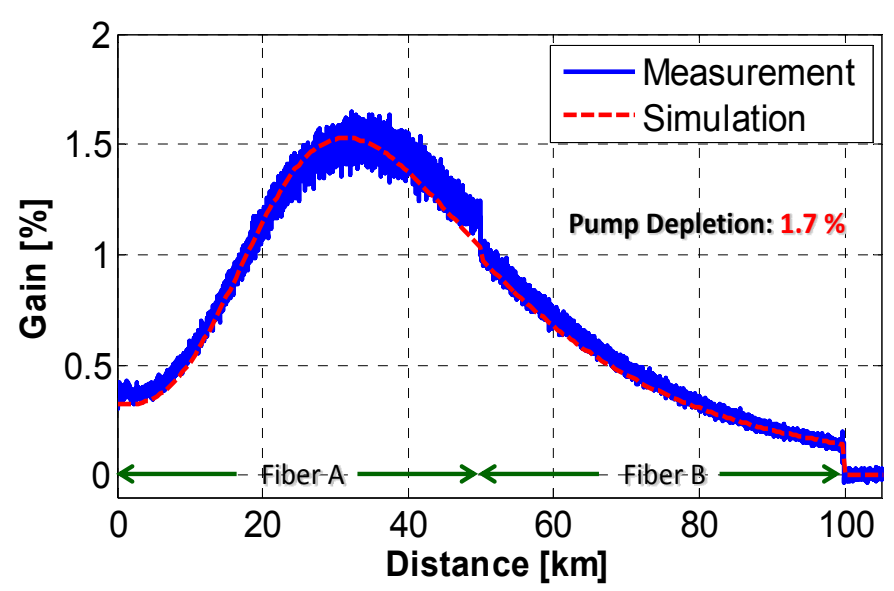

Fig. 4. Raw traces of the optical response of the sensor with single-end distributed Raman amplification. The peak power is shifted at a $30 \mathrm{~km}$ distance in the fiber, extending the sensing range in the same proportion.

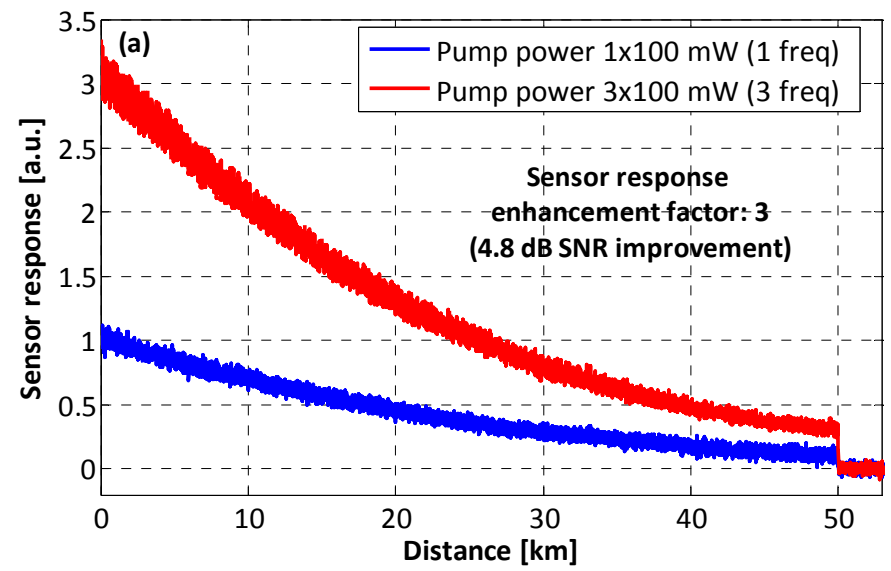

Fig.5. Raw traces of the optical response of the sensor, for a single pulse (blue) and for 3 frequency-multiplexed time-shifted pulses. The SNR gain is $4.8 \mathrm{~dB}$ for an equivalent measuring time.

\section{CONCLUSION}

Making a good Brillouin distributed sensor is not only a matter of a proper assembling of optical devices following a standard scheme. It requires a deep knowledge and an advanced know-how on all nonlinear effects in optical fibers since these interactions are ultimately limiting the response of the sensor. Neglecting side effects such as depletion may lead to a sensing system that apparently shows the expected response, but that will eventually deliver biased evaluations of the measured quantity. But above all, the distinction between a good and a bad sensor is in the perfect control of the noise in the system that is ultimately limiting all specifications of the sensor.

\section{ACKNOWLEDGEMENT}

The authors acknowledge the support from the Swiss Commission for Technology and Innovation (Project 13122.1). This work was performed in the framework and with the support of the COST Action TD1001 OFSeSa.

\section{REFERENCES}

[1] M. A. Soto and L. Thévenaz, "Modeling and evaluating the performance of Brillouin distributed optical fiber sensors," Optics Express, vol. 21, pp. 31347-31366, 2013.

[2] S. M. Foaleng and L. Thevenaz, "Impact of Raman scattering and modulation instability on the performances of Brillouin sensors," in 21st International Conference on Optical Fiber Sensors, Ottawa, Canada, Proc. SPIE 7753, 2011, paper 77539V.

[3] L. Thévenaz, S. Foaleng Mafang, and J. Lin, "Effect of pulse depletion in a Brillouin optical time-domain analysis system," Opt. Express, vol. 21, pp. 14017-14035, 2013 .

[4] A. Minardo, R. Bernini, and L. Zeni, "A Simple Technique for Reducing Pump Depletion in Long-Range Distributed Brillouin Fiber Sensors," IEEE Sensors Journal, vol. 9, pp. 633-634, 2009.

[5] J. Urricelqui, M. Sagues, and A. Loayssa, "BOTDA measurements tolerant to non-local effects by using a phase-modulated probe wave and RF demodulation," Opt. Express, vol. 21, pp. 17186-17194, 2013.

[6] M. A. Soto and L. Thévenaz, "Towards 1'000'000 resolved points in a distributed optical fibre sensor," in 23rd International Conference on Optical Fibre Sensors, Santander, Spain, Proc. SPIE 9157, 2014, paper 9157C3. 
[7] X. Angulo-Vinuesa, S. Martin-Lopez, J. Nuno, P. Corredera, J. D. AniaCastanon, L. Thevenaz, et al., "Raman-Assisted Brillouin Distributed Temperature Sensor over $100 \mathrm{~km}$ featuring 2 meter Resolution and $1.2^{\circ} \mathrm{C}$ Uncertainty," Journal of Lightwave Technology, vol. 30, pp. 1060-1065, 2012.

[8] M. A. Soto, G. Bolognini, F. Di Pasquale, and L. Thévenaz, "Simplexcoded BOTDA fiber sensor with $1 \mathrm{~m}$ spatial resolution over a $50 \mathrm{~km}$ range," Opt. Lett., vol. 35, pp. 259-261, 2010.

[9] F. Rodriguez-Barrios, S. Martin-Lopez, A. Carrasco-Sanz, P. Corredera, J. D. Ania-Castanon, L. Thevenaz, et al., "Distributed Brillouin Fiber Sensor Assisted by First-Order Raman Amplification," Lightwave Technology, Journal of, vol. 28, pp. 2162-2172, 2010.

[10]F. Gyger, E. Rochat, S. Chin, M. Niklès, and L. Thévenaz, "Extending the sensing range of Brillouin optical time-domain analysis up to $325 \mathrm{~km}$ combining four optical repeaters," in 23rd International Conference on Optical Fibre Sensors, Santander, Spain, 2014, Proc. SPIE 9157, paper 91576Q.

[11] M. A. Soto, A. L. Ricchiuti, L. Zhang, D. Barrera, S. Sales, and L. Thévenaz, "Enhanced response in Brillouin distributed optical fibre sensors by simultaneous time and frequency pump multiplexing," in 23rd International Conference on Optical Fibre Sensors, Santander, Spain, Proc. SPIE 9157, 2014, paper 91575T. 